\title{
Low cost liquid crystal display optimized as a monopixel coherent modulator
}

\author{
Andrés Márquez ${ }^{1}$, Mariela L. Álvarez ${ }^{1}$, Luis A. Puerta ${ }^{1}$, Rocío Estévez $^{1}$, Elena Fernández $^{2}$, \\ Augusto Beléndez ${ }^{1}$, Inmaculada Pascual ${ }^{2}$ \\ ${ }^{1}$ Depto. de Física, Ingeniería de Sistemas y Teoría de la Señal, Universidad de Alicante, Spain \\ ${ }^{2}$ Departamento Interuniversitario de Óptica, Universidad de Alicante, Spain \\ Tel.: +34-96-5903400 (ext. 2543); Fax: +34-96-5909750; E-mail: Andres.Marquez@ua.es
}

\begin{abstract}
We propose the use of low cost alphanumeric liquid crystal displays (LCD) not only as an educational tool, to introduce students the insights of the electrooptic performance of liquid crystals, but also as a device able to be used in research to validate models describing the performance of LCDs and to be used in applications requiring monopixel optical modulation. High resolution LCDs have usually focused the research attention because of their capabilities to be used as SLMs. However low cost LCDs with a low number of pixels and directly addressed, such as segmented alphanumeric displays, may also be appealing devices. We show the characterization of one of the segments. The model applied had previously been demonstrated with high resolution LCDs, which are thin LCDs. The segmented LCD is found to be a thick LCD, thus, allowing us to verify the validity of the model in another range of conditions. Furthermore, we show that low cost alphanumeric LCDs can be used as monopixel LCDs. This is interesting since there are a number of applications, as in polarimetry or in interferometry, where monopixel LCDs may proof necessary. We optimize the modulation of the LCD both in the transmissive and in the reflective geometry to obtain amplitude-only and phase-only modulations which are useful for a number of applications.
\end{abstract}

Keywords: Liquid crystal displays, spatial light modulation, electrooptics, transmissive LCD, reflective LCD.

\section{INTRODUCTION}

Nowadays, liquid crystal displays (LCDs) ${ }^{1,2}$ are a well established technology, with a high resolution capability of millions of pixels, widely used in most of the consumer electronics devices which need to display information, such as digital cameras, mobile phones, or TV screens. LCDs have also found widespread use as spatial light modulators (SLMs) in applications such as optical image processing, diffractive optics, holographic data storage, adaptive optics or optical metrology $\mathrm{y}^{3-9}$. In these applications we are interested in using the LCD capability to modify in real time the amplitude, phase and/or the state of polarization of a light beam. LCDs can be used as transmissive or as reflective devices ${ }^{1,2}$. In principle, transmissive LCDs require simpler setups since there is no need to introduce beam-splitters and the LCD can be illuminated perpendicularly. However, reflective LCDs offer approximately twice the dynamic modulation range since the light beam passes twice through the device ${ }^{10}$. This is especially important in applications requiring phase-shift modulation of the incident wavefront. In the last years a reflective LCD technology, the liquid crystal on silicon (LCoS) display, has attracted considerable interest due to some important manufacturing improvements leading in many respects to a superior performance ${ }^{2,11}$.

High resolution LCDs have usually focused the research attention because of their capabilities to be used as SLMs. However low cost LCDs with a low number of pixels, such as segmented alphanumeric displays ${ }^{12,13}$, may also be appealing devices because of the following reasons. First, they are directly addressed, i.e. the electrical signal is directly

Liquid Crystals and Applications in Optics, edited by Milada Glogarova, Peter Palffy-Muhoray, Martin Copic Proc. of SPIE Vol. 6587, 658716, (2007) · 0277-786X/07/\$18 · doi: 10.1117/12.722648 
applied to the electrodes associated to a certain segment. This makes them a very useful device to proof the validity of models describing the electrooptic performance and the modulation capabilities of liquid crystal devices, also valid for high resolution LCDs, without additional effects due to the application of more complex video signals ${ }^{14}$. Second, the size of the segments is big enough so that we can analyze an individual segment with the unexpanded laser beam. In this way the display is used as a monopixel LCD. This is interesting since there are a number of applications, as in polarimetry or in interferometry, where monopixel LCDs may proof necessary. They can be used as variable attenuators, variable retarders, phase-shifters, tunable interference filters, polarization states generator. Furthermore, we can also mention the interest of low cost directly addressed LCDs for educational purposes, as a tool to provide an easier and inexpensive introduction to the electrooptic performance of liquid crystal devices ${ }^{12}$.

To obtain an accurate control of the amplitude and phase of a light beam it is important a proper calibration of the modulation capabilities of the LCD. A series of reverse engineering models, based on the Jones matrix formalism for polarized light, have been proposed ${ }^{15-17}$ to describe the modulation of the anisotropic properties of the LC layer as a function of the applied voltage. Other approaches consider the LCD as a black box and concentrate on the numerical evaluation of the matrix elements of the Jones matrix describing the $\mathrm{LCD}^{18}$. Among the reverse engineering models, the one proposed in Ref. 17 has demonstrated a good accuracy in the prediction of the amplitude and phase-shift modulations for LCDs ${ }^{19,20}$. In principle, this model has been used with commercially available LCDs. Present LCDs have large resolutions, forcing a decrease in the thickness of the device to provide a faster response for the addressing of the pixels $^{1,2}$. In Ref. 19 and 20 it was demonstrated an optimization procedure leading to amplitude-only and phase-only modulations for thin-LCDs. To widen the usefulness of the model it would be interesting to analyze if it also provides accurate complex amplitude predictions for thick LCDs.

In this work we propose the use of low cost alphanumeric LCDs not only as an educational tool, to introduce students the insights of the electrooptic performance of liquid crystals, but also as a device able to be used in research to validate models describing the performance of LCDs and to be used in applications requiring monopixel optical modulation. We show the complex amplitude modulation provided by the alphanumeric LCD both as a transmissive and as a reflective device. This paper continues the work presented in Ref. 13. First, in Section 2 we present the theory and the model we use to characterize and optimize the performance of LCDs. In Section 3 the results for the calibration of the parameters describing the structure and the modulation of the optical anisotropy are provided. We introduce a novel method especially useful for monopixel LCDs to solve the ambiguity in one of the parameters. The method is based on the Fabry-Perot effects occurring in the LCD. In Section 3 we obtain the optimum configurations for amplitude-only or phase-only modulations, both for the transmissive and the reflective geometry. Eventually, the main conclusions of the work are given in Section 5.

\section{THEORETICAL MODEL}

\subsection{Transmissive LCDs}

There are different kinds of LCDs depending on the liquid crystal phase and the cell geometry. In general, commercial LCDs are twisted nematic ${ }^{1,2}$, where twisted means that the optical axis for the molecules twists about $90^{\circ}$ from the entrance to the exit face, and nematic corresponds to the liquid crystal phase employed. To calculate the modulation curves for the LCD we need to know a series of parameters. They can be divided in independent and dependent on the applied voltage $e^{1,17}$. The voltage independent parameters are the total twist angle $(\alpha)$, the orientation of the director axis at the entrance face $\left(\Psi_{\mathrm{D}}\right)$ and the value of the maximum birefringence $\left(\beta_{\max }\right)$. The voltage dependent parameters are related with the profile of the optical anisotropy across the LC layer and its variation with the applied voltage. We use the approximate model in Ref. 17 to describe this profile. This model provides very accurate calculations of amplitude and phase modulation provided by $\operatorname{LCDs}^{18-20}$.

This model takes into account that the molecules in the vicinity of the faces of the LCD are highly anchored to the alignment layer in the windows and can not reorient with the applied voltage ${ }^{1}$. The total thickness of the LCD can be divided in two edge layers with a thickness $d_{1}$ and a central layer with a thickness $d_{2}$, as given in Fig. 1. Thus, the 
anisotropic properties across the LCD are simplified in two voltage dependent parameters, the birefringence in the central layer $(\beta)$ and the birefringence in the edge layers $(\delta)$, which are defined as,

$$
\beta=\pi \Delta n d_{2} / \lambda_{0} ; \delta=\pi \Delta n_{\max } d_{1} / \lambda_{0}
$$

where $\lambda_{0}$ is the wavelength of the light beam, $\Delta n$ is the difference between the extraordinary and ordinary index of refraction, where $\Delta n_{\max }$ is the maximum value. The birefringences depend on the applied voltage through the layer thicknesses $d_{1}(V)$ and $d_{2}(V)$, and the index difference $\Delta n(V)$.

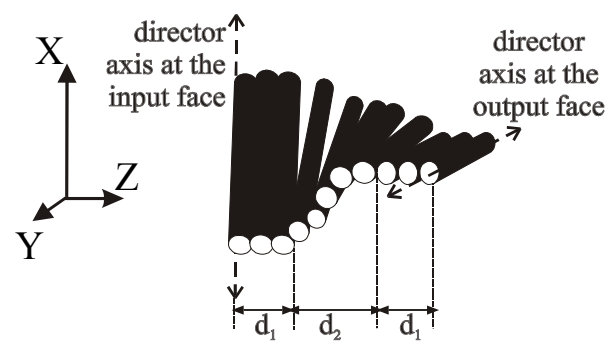

Figure 1. Model for the twisted nematic cell with the two edge layers of thickness $d_{l}$ and the central part of thickness $d_{2}$.

Applying this model and using the Jones matriz formalism for polarized light we obtain the matrix $\mathrm{M}_{\mathrm{LCD}}$ describing the action of the LCD in transmission,

$$
\mathrm{M}_{L C D}=\exp (-j(\beta+2 \delta)) \mathrm{R}(-\alpha)\left(\begin{array}{cc}
X-j Y & Z \\
-Z & X+j Y
\end{array}\right)
$$

where $\mathrm{R}(-\alpha)$ is the rotation matrix and,

$$
\begin{gathered}
X=\cos \gamma \cos (2 \delta)-\frac{\beta}{\gamma} \sin \gamma \sin (2 \delta) \\
Y=\cos \gamma \sin (2 \delta)+\frac{\beta}{\gamma} \sin \gamma \cos (2 \delta) \\
Z=\frac{\alpha}{\gamma} \sin \gamma \\
\gamma=\sqrt{\alpha^{2}+\beta^{2}}
\end{gathered}
$$

The expression in Eq. (2) considers the reference system with its $\mathrm{X}$-axis parallel to the director axis at the entrance face of the LCD.

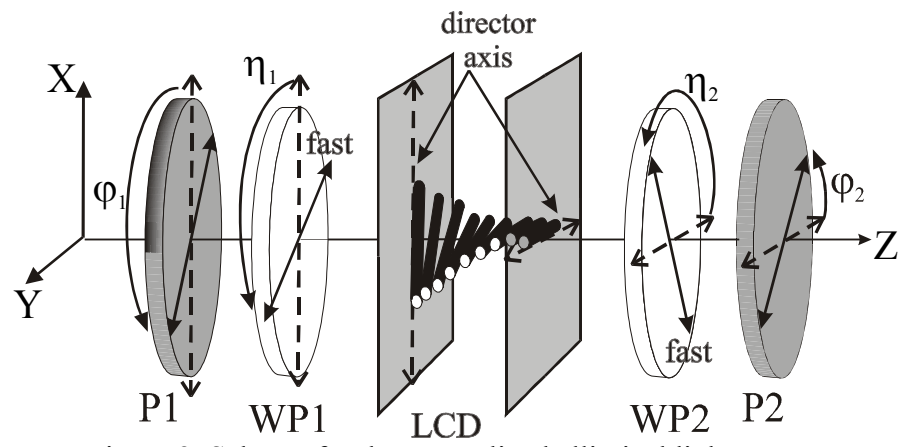

Figure 2. Scheme for the generalized elliptical light set-up. 
Using this model we can calculate, through an optimization procedure, the orientation of the polarizing devices in front and behind the LCD to obtain useful modulation regimes, as amplitude-only or phase-only ${ }^{19,20}$. In general with present LCDs, which are thin devices, we need to use elliptically polarized light to obtain amplitude-only and, especially, phaseonly modulations ${ }^{19,20}$. In the case of phase-only modulation we also need short wavelengths to achieve a $360^{\circ}$ phase range. The setup for elliptically polarized light generation and detection is illustrated in Fig. 2. The wave plates WP1 and WP2 introduce retardances of $\Gamma_{1}$ and $\Gamma_{2}$ respectively. The angles of the elements in front of the LCD are referred to the orientation of the director at the input surface (which we suppose that coincides with the $X$-coordinate axis) while the angles of the elements behind the LCD are measured with respect to the orientation of the director at the output surface.

\subsection{Reflective LCDs}

Using the Jones matrix $\mathrm{M}_{\mathrm{LCD}}$ for a transmissive LCD it can also be calculated the modulation curves provided by a reflective LCD. To this goal we consider the reflective LCD as two transmissive LCDs in cascade, being the first one traversed in the forward direction and the second one, after the reflection at the mirror, in the backward direction. To obtain the Jones matrix for the reflective LCD we consider that the reference system for both the forward and the backward direction is a right handed system. We also consider that the LCD is a non-absorbing reciprocal polarization device $^{12}$. Then the Jones matrix for the reflective LCD is given by the following matrix product,

$$
M_{R}=J M_{L C D}^{t} M_{L C D}
$$

where $\mathrm{M}_{\mathrm{LCD}}^{\mathrm{t}}$ is the transposed of the Jones matrix $\mathrm{M}_{\mathrm{LCD}}$ in the forward direction, and $\mathrm{J}$ is the inversion matrix along the Y-axis. In Fig. 3 we show the geometry in reflection used in this work for the LCD. It is inserted between two polarizers and a mirror. The beam-splitter cube separates the incident from the reflected beam. The addition of the beam splitter allows for normal illumination of the display. In this system the electric field at the output can be obtained as,

$$
\vec{E}_{\text {out }}=\mathrm{P}_{\mathrm{X}} \mathrm{R}\left(\varphi_{2}\right) \mathrm{JM}_{\mathrm{R}}\left(\begin{array}{c}
\cos \varphi_{1} \\
\sin \varphi_{1}
\end{array}\right)
$$

where the inversion matrix $J$ corresponds to the mirror, $P_{X}$ is the Jones matrix for the polarizer, and $R(\omega)$ is a rotation matrix. In Eq. (8) we consider that the beam-splitter cube is an ideal non-polarizing cube, thus the state of polarization (SOP) of the ligh beam transmitted or reflected is not changed by the cube. In many situations the polarization effects of the cube can be actually ignored.

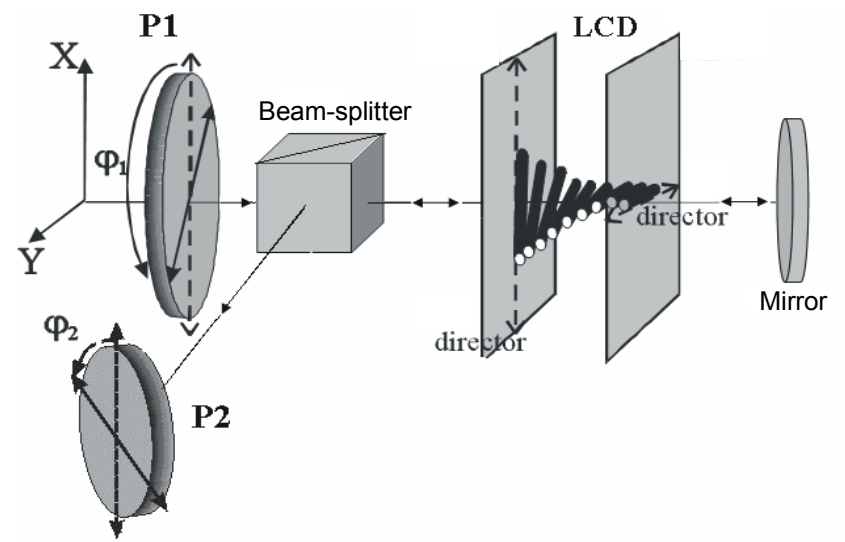

Figure 3. Experimental setup used in this work to use the LCD in a reflective geometry. 


\section{CHARACTERIZATION OF THE LIQUID CRYSTAL DEVICE}

\subsection{Alphanumeric LCD}

In this work we use an alphanumeric LCD, model C-2630, distributed by CEBEK, that we show in Fig. 4(a). We eliminate two sheet polarizers and a silvered sheet (acting as a mirror) attached to the windows of the display, so that the LCD becomes totally transparent, Fig. 4(b). The display has 32 segments (or pixels) shown in Fig. 4(c), and we choose one of them for the measurements in this work. In particular we have chosen the segment corresponding to "overflow" ( $\mathrm{Z}$ in Fig. 4(c)). The display has a common electrode in one of the faces and independent electrodes for each of the segments at the other face. The different electrodes are connected to independent electrical terminations, thus a certain segment can be directly connected to a signal generator. The voltage to be applied must be a bipolar signal with no DC term to avoid the electrolytic degradation of the LC and the electrodes ${ }^{1}$.

(a)

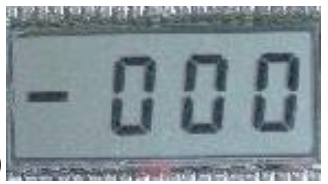

(b)

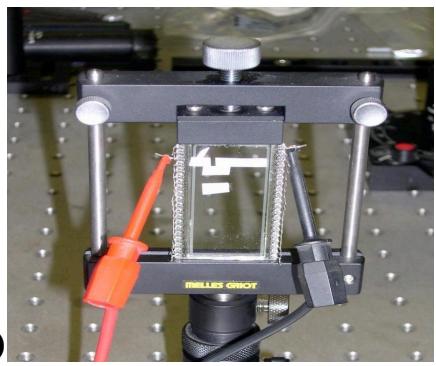

(c)

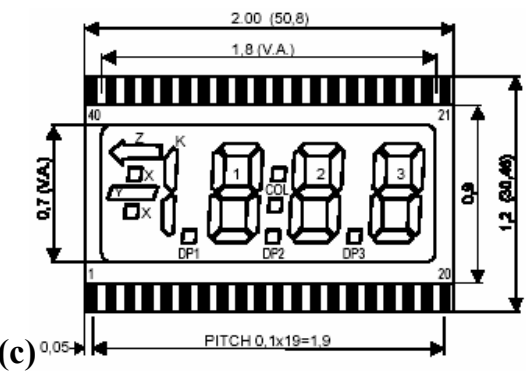

Figure 4. Alphanumeric LC display used in this work. (a) Display in normal operation where the segments can be seen. (b) In the optical setup, totally transparent after removing the sheet polarizers and the mirrored sheet. (c) Schematic diagram with the numbering of the different segments, and the lengths of the different elements in inches (in parentheses in $\mathrm{mm}$ ).

\subsection{Voltage independent parameters}

The voltage independent parameters have been calculated by means of known techniques ${ }^{17,22,23}$. For these measurements we have used the unexpanded beams for three different wavelengths: $633 \mathrm{~nm}$ and $594 \mathrm{~nm}$ from two He-Ne lasers, and 532 from a Nd: $\mathrm{YVO}_{4}$ laser. In Table 1 we summarize the values obtained for the voltage independent parameters. We show two values for the parameter $\Psi_{\mathrm{D}}$. The techniques used to characterize the voltage independent parameters produce identical results for a value of $\Psi_{\mathrm{D}}$ and its perpendicular orientation. To solve this ambiguity we usually apply the technique proposed in Ref. 24. However, it can only be used with pixelated LCDs with a large number of pixels. In Section 3.3 we demonstrate a novel technique to solve this ambiguity with monopixel LCDs.

Table 1.Values of the parameters for the LCD used in the experiments.

\begin{tabular}{|c|c|c|c|c|}
\hline$\alpha\left(^{\circ}\right)$ & $\Psi_{\mathrm{D}}\left({ }^{\circ}\right)$ & $\beta_{\max }(\lambda=633 \mathrm{~nm})$ & $\beta_{\max }(\lambda=594 \mathrm{~nm})$ & $\beta_{\max }(\lambda=532 \mathrm{~nm})$ \\
\hline+95 & +47 or $-43^{\circ}$ & $288^{\circ}$ & $312^{\circ}$ & $360^{\circ}$ \\
\hline
\end{tabular}

In Table 1 the twist angle $\alpha$ is close to $90^{\circ}$. This indicates that the device is a twisted-nematic $\mathrm{LCD}^{1}$, which is the usual configuration in high resolution LCDs. In the case of low resolution displays it is also very common to use the supertwisted-nematic (STN) configuration. The $\beta_{\max }$ values obtained are very high, from $288^{\circ}$ to $360^{\circ}$, depending on the wavelength. This is twice the typical value found in high resolution LCDs. Therefore, the alphanumeric LCD used in this work is actually a thick LCD. This allows us to verify if the model presented in Section 2.1 is valid for thick LCDs. Furthermore, a thick LCD provides a larger dynamic modulation range, which is especially interesting for phase-shift modulation applications. The ratio between between the different $\beta_{\max }$ values, $\left(\beta_{\max (594 \mathrm{~nm})} / \beta_{\max (633 \mathrm{~nm})}\right)=1.08$ and $\left(\beta_{\max (532 \mathrm{~nm})} / \beta_{\max (633 \mathrm{~nm})}\right)=1.25$, is very similar to the values given in the literature ${ }^{17,20}$ for high resolution LCDs. This permits us to say that even though high resolution LCDs are technologically much more advanced, at a fundamental level the electrooptical properties are very similar to the ones exhibited by segmented LC devices. 


\subsection{Method based on the Fabry-Perot effects to measure the orientation of the director at the entrance face}

To solve the ambiguity for the orientation of the director axis at the entrance face $\Psi_{\mathrm{D}}$ we propose an experiment which takes advantage of the Fabry-Perot effects produced by the multiple reflections at the interfaces of the LCD ${ }^{25}$. The origin for these multiple reflections is schematically represented in Fig. 5. When a voltage is applied the LC molecules tilt to align with the direction of the applied electric field. The index ellipsoid associated with the extraordinary and ordinary indices of refraction of the LC molecules reorient. Therefore the optical path length for the light beam traversing the LC layer changes with the voltage, the condition for the interferences between the multiple beams changes, and this produces the fluctuation of the intensity transmitted by the LCD as a function of the voltage. If the interference is purely constructive (destructive) the intensity transmitted by the cell is a maximum (minimum). The maximum optical path length variation with the voltage occurs for a light beam with its electrical field parallel to the optical axis, i.e. parallel to the director axis of the molecules at the entrance face. The ordinary index of refraction does not change with the reorientation of the ellipsoid. Thus, for a light beam with its electrical field perpendicular to the director axis at the input face the oscillations in the transmitted intensity would be minimized.

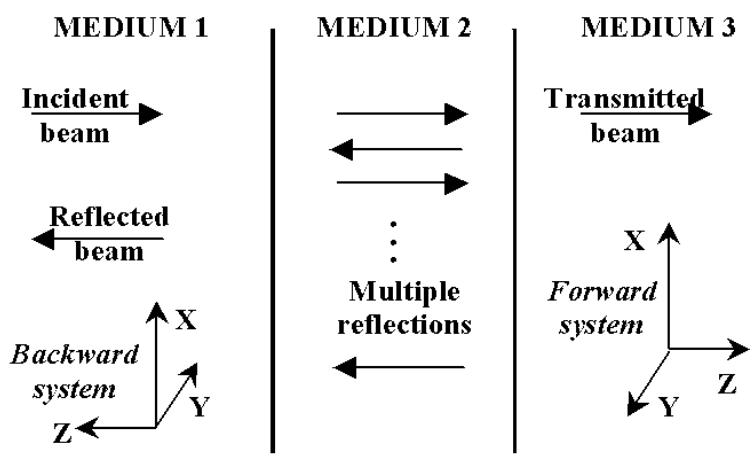

Figure 5. Schematic diagram for the multiple reflections produced at the interfaces for light traversing different media.

To perform the experiment we switch on the LCD for the first time in this work. The voltage signal applied to the LCD is a bipolar signal with no DC component as commented previously. The amplitude of the applied voltage is varied between 0 and $10 \mathrm{~V}$. The parameter of interest is the root mean square (rms) value of the applied voltage since the response of the LC molecules is not affected by the sign of the voltage. We apply a signal with a frequency of $1 \mathrm{kHz}$. LC molecules are very slow to react and at this high frequency value their orientation is not affected by the transition time in the bipolar signal. The signal generator used in this work is a Promax, model GF-232.
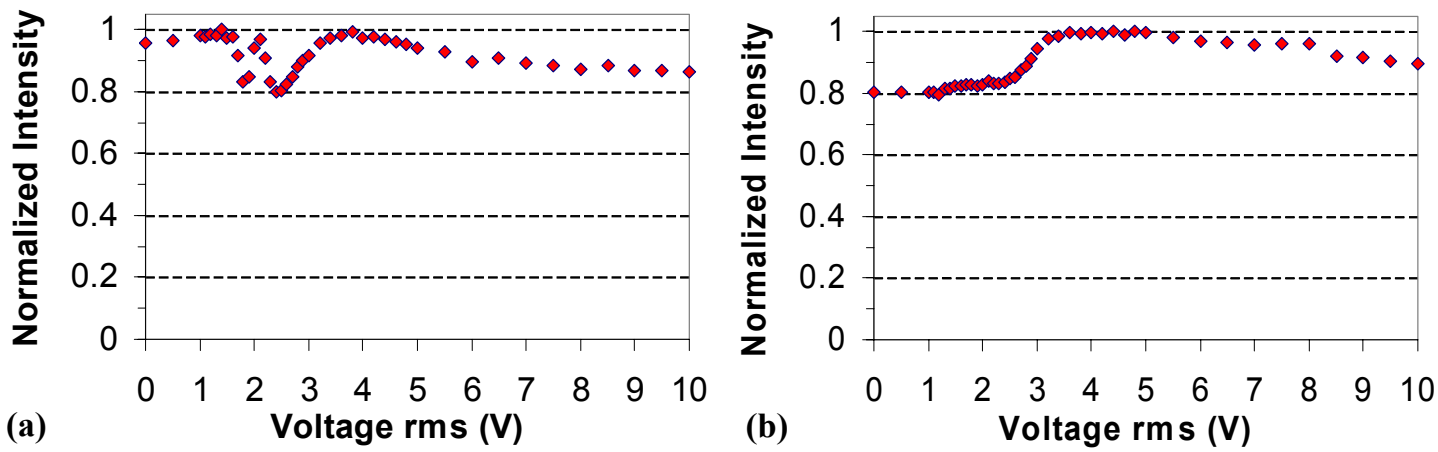

Figure 6. Normalized intensity measured removing the analyzer and with the transmission axis of the input polarizer at an angle (with respect to the vertical of the lab): (a) $+47^{\circ}$. (b) $-43^{\circ}$. Measurements for $\lambda=594 \mathrm{~nm}$. 
In the experiment a linear polarizer is located in front of the LCD and no analyzer is considered. We consider the input polarizer oriented at each of the two angles for $\Psi_{\mathrm{D}},+47^{\circ}$ and $-43^{\circ}$, given in Table 1. For each position we measure the intensity transmitted by the LCD as a function of the applied rms voltage. In Fig. 6(a) and (b) we show respectively the normalized intensity for light incident at $+47^{\circ}$ and $-43^{\circ}$ with respect to the vertical lab. We have used light with a wavelength of $594 \mathrm{~nm}$. We see that the intensity exhibits more fluctuations when the transmission axis of the input polarizer is oriented at $47^{\circ}$, especially in the range between 1 and 5 volts. Therefore, from this experiment we obtain that the orientation of the director axis at the input face is $\Psi_{D}=+47^{\circ}$. The fluctuation in Fig. $6(\mathrm{~b})$ is produced by a percentage of light that decouples from the helix described by the ordinary axis of the molecules across the cell and vibrates along the extraordinary axis.

\subsection{Voltage dependent parameters}

The voltage dependent parameters $\beta(V)$ and $\delta(V)$ are calculated using the technique proposed in Ref. 17. We insert the LCD between two linear polarizers with their transmission axes in specific orientations. The applied voltage is varied in the interval from 0 to $10 \mathrm{~V}$, in steps of 0.1 and $0.2 \mathrm{~V}$ in the most sensitive regions, and in steps of $0.5 \mathrm{~V}$ in the regions with a slower intensity variation. The nonlinear curve fitting of intensity transmission measurements obtained for the three wavelengths versus the applied voltage provide us with the values for $\beta(V)$ and $\delta(V)$.

In Fig. 7 we plot the fitted intensities between the experimental and the theoretical values for the three wavelengths and for the three polarizer configurations used. The value for the orientation of the transmission axis for the input and output polarizer, introduced in Fig. 2, is given between parentheses as $\left(\varphi_{1}, \varphi_{2}\right)$ in the legend of the graphs. The experimental values are represented by the symbols and the fitting by the continuous lines. We note that there is an excellent agreement between theory and experiment. Therefore the model used in this work is perfectly valid for thick LCDs.

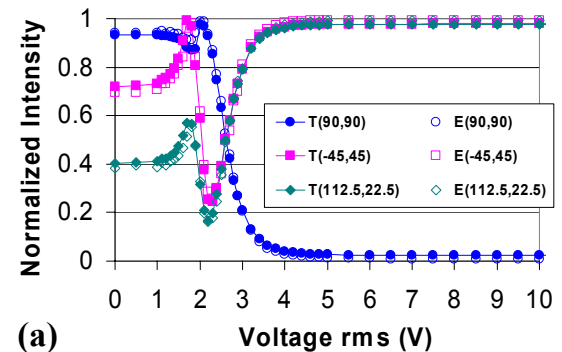

(a)

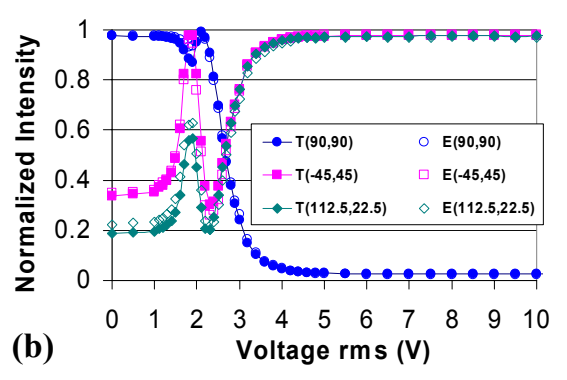

(b)

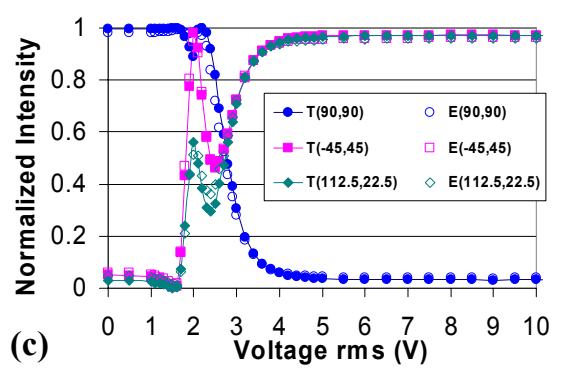

Figure 7. Normalized intensity for the three configurations of polarizers used in the experiments and for the three wavelengths. (a) $\lambda=633 \mathrm{~nm}$, (b) $\lambda=594 \mathrm{~nm}$ and (c) $\lambda=532 \mathrm{~nm}$. Experimental values (symbols) and theoretical fit (continuous lines).

In Fig. 8(a) and (b) we show respectively the values obtained for the birefringences $\beta(V)$ and $\delta(V)$ at the three wavelengths. We observe that the voltage threshold ${ }^{1}$ for the LCD is about $1 \mathrm{~V}$. Below this value the LC molecules do not react and the birefringences exhibit the same value as when the LCD is switched off. The maximum variations are produced in the interval between 1.5 and $4 \mathrm{~V}$. From $5 \mathrm{~V}$ there are almost no further variations, and the LC molecules have attained their maximum angle of tilt. The values for the birefringences between the three wavelengths do not vary independently ${ }^{17}$. We scale them using the ratios obtained between the $\beta_{\max }$ values in Table 1 . The $\beta$ birefringence (Fig. $8(\mathrm{a}))$ decreases monotonically with the increase in the applied voltage. The $\delta$ birefringence (Fig. 8(b)) is zero at low voltages, attains a maximum value at $3 \mathrm{~V}$ and then decreases to a value of $12^{\circ}$ from $4 \mathrm{~V}$. In previous works the $\delta$ birefringence increased monotonically with the voltage. From the model in Ref. 17 it makes sense that the $\delta$ birefringence is close to zero at low values of applied voltage since at this range the edge layers disappear. The result at high voltage needs further discussion. A more rigorous interpretation of the model is compatible with the decrease of the $\delta$ birefringence at high voltage, as discussed in Ref. 26. It will be demonstrated in Section 4 that the values for the birefringences in Fig. 8 are able to predict with a high accuracy the amplitude and phase modulation curves for the LCD. 

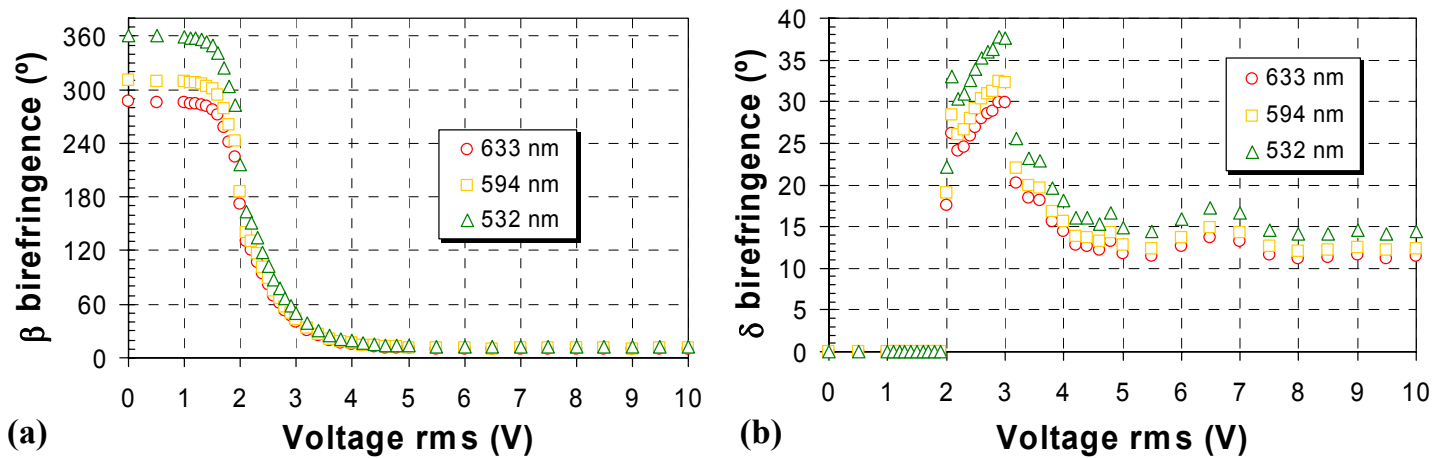

Figure 8. Values obtained for the voltage dependent parameters at the three wavelengths. (a) $\beta(V)$, (b) $\delta(V)$.

\section{OPTIMUM CURVES FOR OPTICAL MODULATION}

In what follows we show how applying the model in Section 2, which makes use of the characterization results provided in Section 3, and applying a computer search for the orientation of the external polarization elements we are able to find amplitude-only or phase-only configurations, useful for a number of applications. We consider the LCD both in the transmissive and in the reflective geometries given respectively in Fig. 2 and 3. The light beam considered in the experiments has a wavelength of $633 \mathrm{~nm}$. In the transmissive setup, first we analyze configurations with only polarizers, and then including the wave plates to generate and detect elliptical polarized light. In the reflective geometry we only consider linear polarizers. At $633 \mathrm{~nm}$ the retardances measured for the two wave plates (Fig. 2) are $\Gamma_{l}=73$ degrees and $\Gamma_{2}=73$ degrees.

In the most general case when wave plates and polarizers are included in the setup we have four magnitudes, $\eta_{1}, \eta_{2}, \varphi_{1}$ and $\varphi_{2}$, left as free parameters in the optimization process. We assign initial arbitrary values to the angles $\eta_{1}, \eta_{2}, \varphi_{1}$ and $\varphi_{2}$, and we calculate the transmittance $T$ and the phase-shift $\psi$ as a function of the gray level. The optimization criterion to be applied depends on the modulation regime we are interested on. In the amplitude-only regime we look for a maximum intensity contrast and a minimum phase-shift modulation depth. In the phase-only regime we look for a constant intensity and a phase-shift modulation depth of $360^{\circ}$. In some of the graphs that we present in this Section we have measured the experimental values to verify the accuracy of the results provided by the theory. In these graphs the experimental data are plotted as circles and the theoretical prediction as continuous lines.
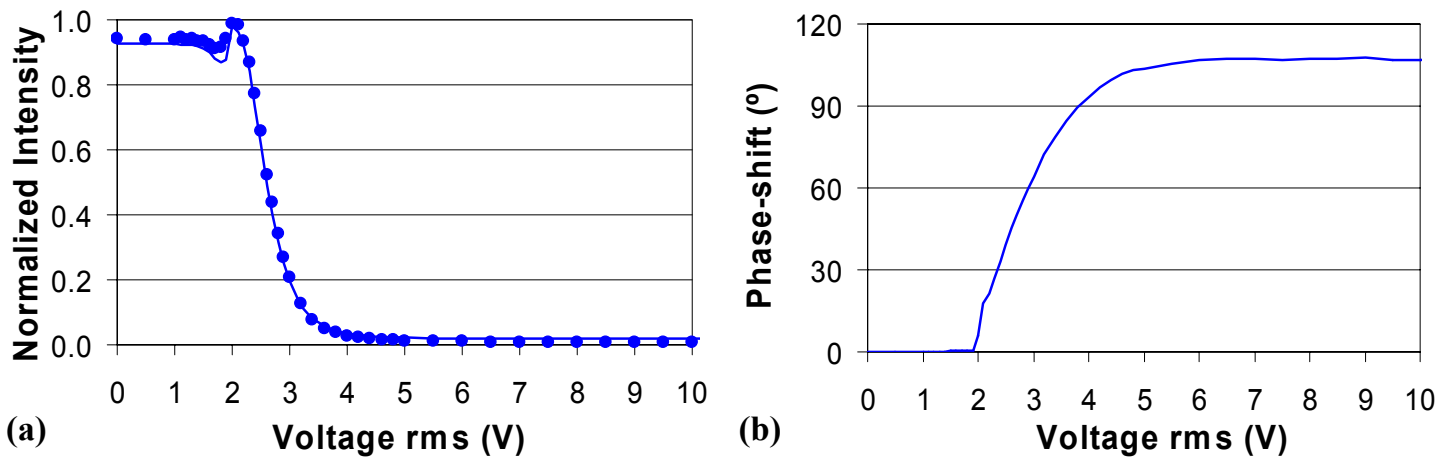

Figure 9. Amplitude-only modulation with only polarizers $\left(\varphi_{1}=90^{\circ}, \varphi_{2}=90^{\circ}\right)(\lambda=633 \mathrm{~nm})$. (a) Normalized intensity. (b) Phase-shift. The symbols correspond to experimental measurements and the lines correspond to predicted values.

Next, we show the results for the amplitude-only regime in the transmissive geometry. In Fig. 9(a) and 9(b) we show respectively the normalized intensity and the phase-shift curves as a function of the applied voltage considering only linear polarizers. The orientation for the transmission axis of the polarizers is given in the caption of the figure. Their 
transmission axes are perpendicular to the director axis at the entrance and exit faces respectively. The light is thus mainly affected by the ordinary index of refraction, which does not change with the applied voltage. This minimizes the phase-shift modulation depth. Strictly speaking we would say that this is an amplitude-mostly regime since the phaseshift modulation depth is not exactly zero. The intensity curve has a very good contrast with a maximum value close to one and a minimum very close to zero. In principle the interval of interest for amplitude-only modulation is in the range from 2 to 5 V. In Fig. 9(a) we observe the excellent agreement between experiment and theory.

In principle, using elliptically polarized light we can obtain a configuration with a reduced phase-modulation depth. In Fig. 10(a) and 10(b) we show respectively the normalized intensity and the phase-shift curves considering polarizers and wave plates. Now the phase-shift depth is halved in comparison with Fig. 9(b). The intensity profile is very similar to previous Fig. 9(a). Actually, the orientation of polarizers and wave plates (see the caption) produces light with a very low ellipticity and almost parallel to the orientation of the polarizers in Fig. 9. Once again, in Fig. 10(a) we observe a good agreement between experiment and theory. A monopixel LCD in the amplitude-only regime can be used as a variable attenuator, or as a switch if we are just interested in two transmittance levels.
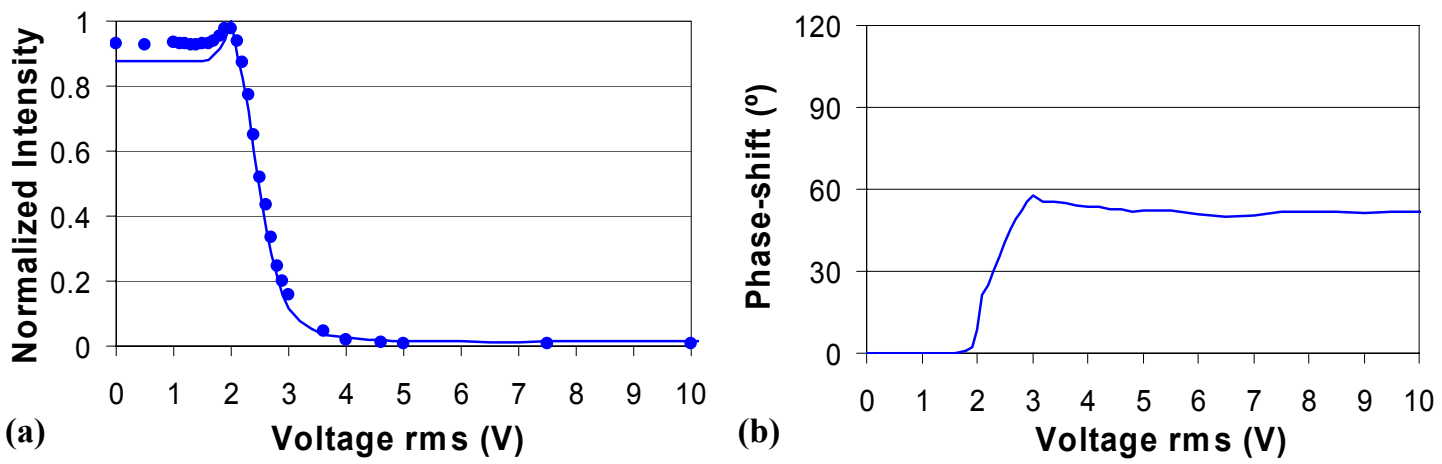

Figure 10. Amplitude-only modulation with elliptical light $\left(\varphi_{1}=85^{\circ}, \varphi_{2}=88^{\circ}, \eta_{1}=0^{\circ}, \eta_{2}=80^{\circ}\right)(\lambda=633 \mathrm{~nm})$. (a) Normalized intensity. (b) Phase-shift. The symbols correspond to experimental measurements and the lines correspond to predicted values.

In Fig. 11 and 12 we show the results for the phase-only regime in the transmissive geometry. In these configurations we have measured experimentally the phase-shift to verify the accuracy of the theoretical predictions. To this goal we have used a Mach-Zehnder interferometer. In Fig. 11(a) and 11(b) we show respectively the normalized intensity and the phase-shift curves with only-polarizers. The phase-shift covers the $360^{\circ}$ range. However, the intensity has noticeable fluctuations in the low voltage region which is the interval where most of the phase-shift modulation is produced. This is not a good phase-only configuration, even though this depends on the requirements of the application. We note that the agreement between theory and experiment is very good.
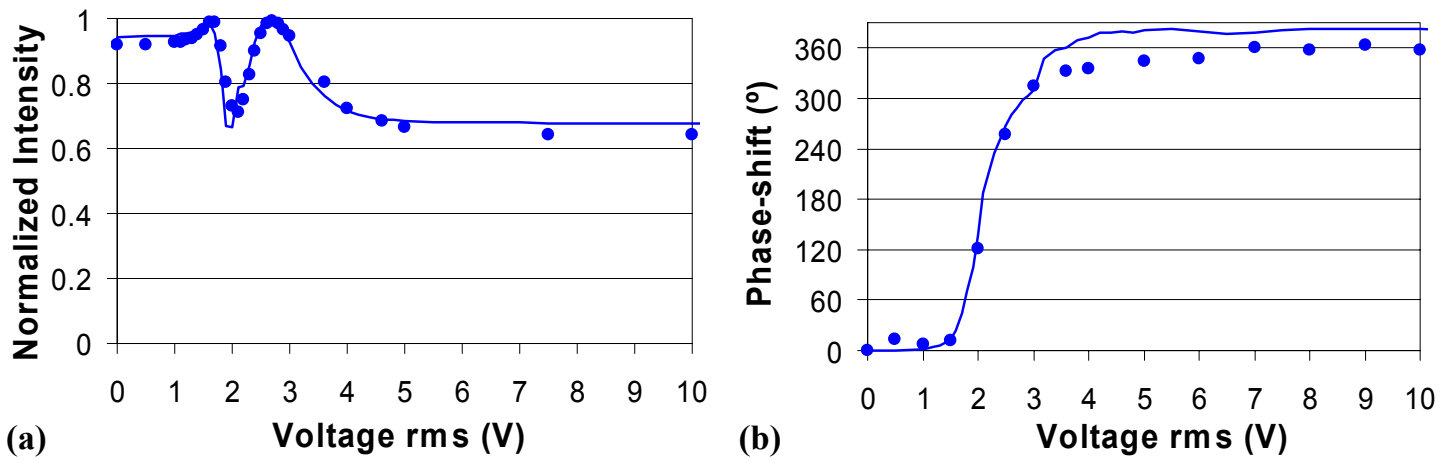

Figure 11. Phase-only modulation with only polarizers $\left(\varphi_{I}=-28^{\circ}, \varphi_{2}=20^{\circ}\right)(\lambda=633 \mathrm{~nm})$. (a) Normalized intensity. (b) Phaseshift. The symbols correspond to experimental measurements and the lines correspond to predicted values. 
In Fig. 12(a) and 12(b) we show respectively the normalized intensity and the phase-shift curves including the wave plates. We see that the phase-shift depth is significantly larger than previously. The use of elliptically polarized light is also responsible for the more constant intensity transmission. In principle the voltage interval of interest would be between about 2 and $4 \mathrm{~V}$. The agreement between theory and experiment is good as in the previous cases. The differences in Fig. 12(a) may be produced by the error of $\pm 1^{\circ}$ that we have in the orientation of the wave plates and polarizers $^{27}$. A monopixel LCD in the phase-only regime can be used as a phase-shifter useful in interferometric setups for metrology. We note that in this work we are able to obtain phase-only modulation at $633 \mathrm{~nm}$ with our thick LCD. With high resolution LCDs, which are thin devices, we should use short wavelengths (blue-violet) to obtain enough phase-shift modulation depth.
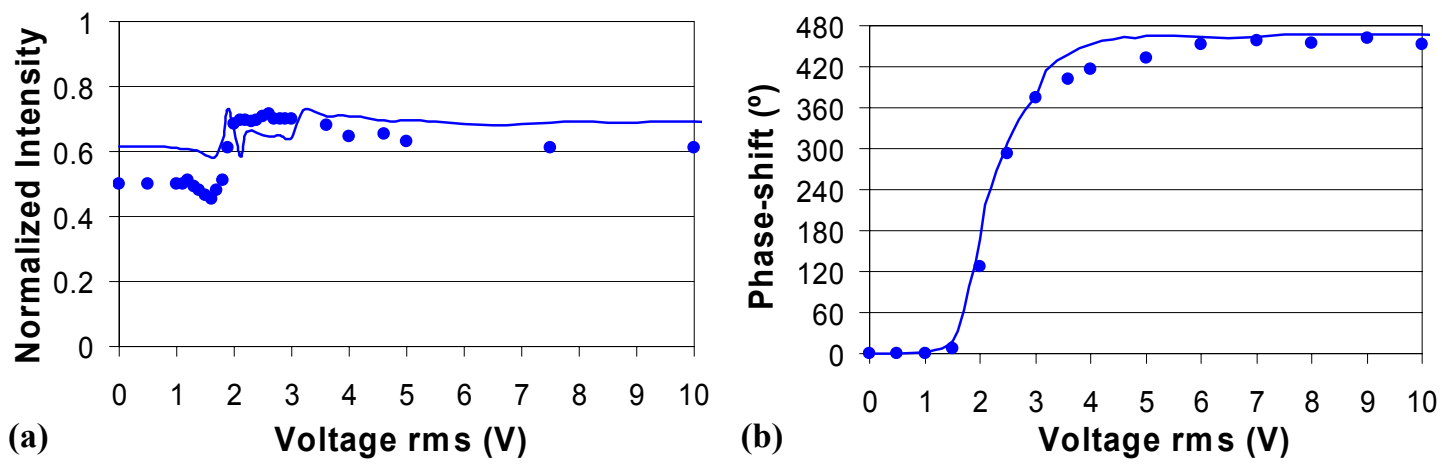

Figure 12. Phase-only modulation with elliptical light $\left(\varphi_{1}=38^{\circ}, \varphi_{2}=22^{\circ}, \eta_{1}=0^{\circ}, \eta_{2}=92^{\circ}\right)(\lambda=633 \mathrm{~nm})$. (a) Normalized intensity. (b) Phase-shift. The symbols correspond to experimental measurements and the lines correspond to predicted values.

Next, we want to verify if the characterization performed in Section 3 is useful to calculate the modulation of the LCD in the reflective geometry. In Fig. 13(a) and 13(b) we show respectively the normalized intensity and the phase-shift curves for phase-only modulation with only-polarizers. We note that the phase shift has actually doubled in comparison with the values obtained in the phase-only configurations in the transmissive geometry. The intensity is very constant with a $20 \%$ decrease in the low voltage region. The voltage interval of interest would be between about 2 and $3.2 \mathrm{~V}$, where the intensity stays constant and the phase-shift covers $360^{\circ}$. In the reflective geometry we do not need the addition of wave plates to obtain phase-only modulation. The agreement between theory and experiment in Fig. 13(a) is very good. Therefore the values in Section 3 can be used to calculate the performance of the LCD in the reflective geometry.
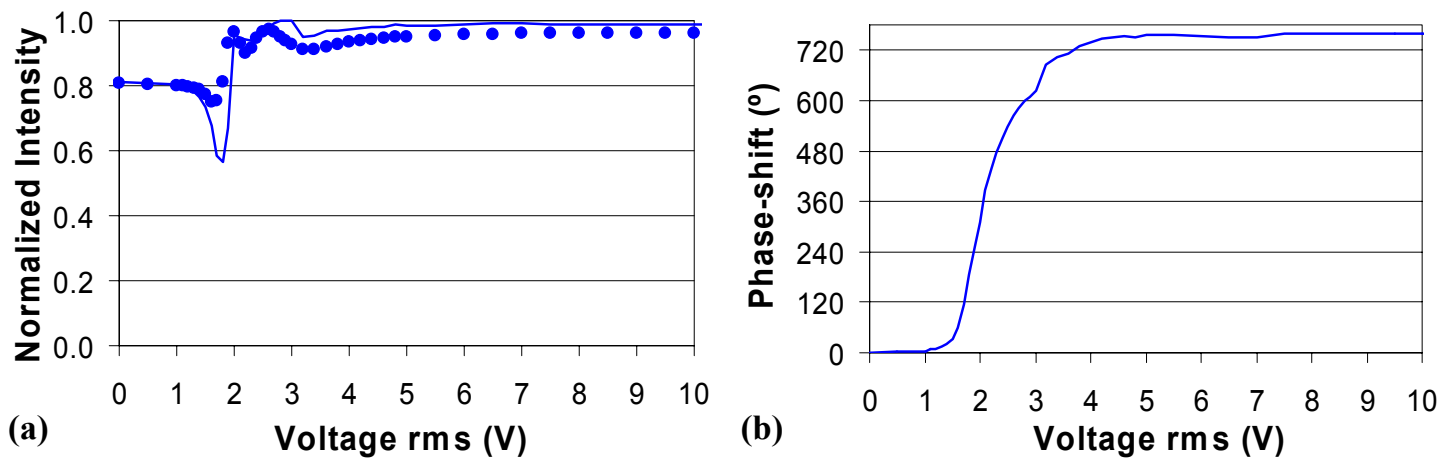

Figure 13. Reflective geometry. Phase-only modulation with only polarizers $\left(\varphi_{1}=0^{\circ}, \varphi_{2}=0^{\circ}\right)(\lambda=633 \mathrm{~nm})$. (a) Normalized intensity. (b) Phase-shift. The symbols correspond to experimental measurements and the lines correspond to predicted values. 


\section{CONCLUSIONS}

We have demonstrated the interest of low cost alphanumeric liquid crystal displays (LCD) not only as an educational tool but also as a device able to be used in research to validate models describing the performance of LCDs and to be used in applications requiring monopixel optical modulation. We have characterized one of the segments. The segmented LCD is found to be a thick LCD. This has allowed us to verify the validity of the model proposed in Ref. 17 in another range of conditions. To solve the ambiguity in the orientation of the director axis at the entrance face we have proposed a technique based on the Fabry-Perot interferences produced by the multiple reflections at the interfaces of the LCD. We have optimized the modulation of the LCD both in the transmissive and in the reflective geometry to obtain amplitudeonly and phase-only modulation configurations both with only polarizers and with elliptically polarized light. We have demonstrated that low cost alphanumeric LCDs are attractive and reliable devices to be used as monopixel optical modulators.

\section{ACKNOWLEDGMENTS}

This work was supported by the Spanish Ministerio de Educación y Ciencia (grants FIS2005-05881-C02-01 and FIS2005-05881-C02-02), and from Generalitat Valenciana (grant GV06-007).

\section{REFERENCES}

1. P. Yeh and C. Gu, Optics of liquid crystal displays, John Wiley \& Sons, (1999).

2. T. Wu and D. K. Yang, Reflective Liquid Crystal Displays, John Wiley \& Sons Inc., (2005).

3. H. K. Liu, J. A. Davis and R. A. Lilly, "Optical-data-processing properties of a liquid-crystal television spatial light modulator", Opt. Lett. 10, 635-637 (1985).

4. V. Laude, "Twisted-nematic liquid-crystal pixelated active lens", Opt. Commun. 153, 134-152 (1998).

5. J. A. Davis, J. C. Escalera, J. Campos, A. Márquez, M. J. Yzuel and C. Iemmi, "Programmable axial apodizing and hyperresolving amplitude filters using a liquid crystal spatial light modulator", Opt. Lett. 24, 628-630 (1999).

6. A.Márquez, C. Iemmi, J. Campos and M. J. Yzuel, "Achromatic diffractive lens written onto a liquid crystal display", Opt. Lett. 31, 392-394 (2006).

7. H.J. Coufal, D. Psaltis and B.T. Sincerbox, eds., Holographic Data Storage, Springer-Verlag, Berlin, (2000).

8. Q. Mu, Z. Cao, L. Hu, D. Li and L. Xuan, "Adaptive optics imaging system based on a high resolution liquid crystal on silicon device", Opt. Express 14, 8013-8018 (2006).

9. W. Osten, C. Kohler and J. Liesener, "Evaluation and application of spatial light modulators for optical metrology", Opt. Pura Apl. 38 (3), 71-81 (2005).

10. I. Moreno, C. R. Fernández-Pousa, J. A. Davis and D. J. Franich, "Polarization eigenvectors for reflective twisted nematic liquid crystal displays", Opt. Eng. 40, 2220-2226 (2001).

11. Y. Lee, J. Gourlay, W. J. Hossack, I. Underwood and A. J. Walton, "Multi-phase modulation for nematic liquid crystal on silicon backplane spatial light modulators using pulse-width modulation driving scheme," Opt. Comm. 236, 313-322 (2004).

12. S. Ledesma, H. Lozza and M. Pomata, "Undergraduate optical experiments using simple liquid crystal displays", Proc. SPIE 3831, 342-351 (2000).

13. M. L. Álvarez, A. Márquez, L. A. Puerta, R. Estévez, E. Fernández, A. Beléndez and I. Pascual, "Caracterización de una pantalla de cristal líquido de baja resolución para su uso como modulador óptico", Opt. Pura Apl. 40, 95-103 (2007).

14. A.Márquez, C. Iemmi, I. Moreno, J. Campos and M. J. Yzuel, "Anamorphic and spatial frequency dependent phase modulation on liquid crystal displays. Optimization of the modulation diffraction efficiency," Opt. Express 13, 2111-2119 (2005).

15. K. Lu and B. E. A. Saleh, "Theory and design of the liquid crystal TV as an optical spatial phase modulator", Opt. Eng. 29, 240-246 (1990).

16. J. A. Coy, M. Zaldarriaga, D. F. Grosz and O. E. Martinez, "Characterization of a liquid crystal television as a programable spatial light modulator”, Opt. Eng. 35, 15-19 (1996). 
17. A. Márquez, J. Campos, M. J. Yzuel, I. Moreno, J. A. Davis, C. Iemmi, A. Moreno and A. Robert, "Characterization of edge effects in twisted nematic liquid crystal spatial light modulators", Opt. Eng. 39, 3301-3307 (2000).

18. I. Moreno, P. Velásquez, C. R. Fernández-Pousa, M. M. Sánchez-López and F. Mateos, "Jones matrix method for predicting and optimizing the optical modulation properties of a liquid-crystal display", J. Appl. Phys. 94, 3697 (2003).

19. A. Márquez, C. Iemmi, I. Moreno, J. A. Davis, J. Campos and M. J. Yzuel, "Quantitative prediction of the modulation behavior of twisted nematic liquid crystal displays based on a simple physical model," Opt. Eng. 40, 2558-2564 (2001).

20. J. Nicolás, J. Campos and M. J. Yzuel, "Phase and amplitude modulation of elliptic polarization states by nonabsorbing anisotropic elements: application to liquid crystal devices," J. Opt. Soc. Am. A 19, 1013-1020 (2002).

21. C. R. Fernández-Pousa, I. Moreno, N. Bennis and C. Gómez-Reino, "Generalized formulation and symmetry properties of reciprocal nonabsorbing polarization devices: application to liquid-crystal displays", J. Opt. Soc. Am. A $17,2074-2080(2000)$.

22. C. Soutar and K. Lu, "Determination of the physical properties of an arbitrary twisted-nematic liquid crystal cell", Opt. Eng. 33, 2704-2712 (1994).

23. J. A. Davis, D. B. Allison, K. G. D'Nelly, M. L. Wilson and I. Moreno, "Ambiguities in measuring the physical parameters for twisted-nematic liquid crystal spatial light modulators", Opt. Eng. 38, 705-709 (1999).

24. J. A. Davis, P. Tsai, K. G. D'Nelly and I. Moreno, "Simple technique for determining the extraordinary axis direction for twisted-nematic liquid crystal spatial light modulators", Opt. Eng. 38, 929-932 (1999).

25. A. Márquez, I. Moreno, J. Campos, M. J. Yzuel, "Analysis of Fabry-Perot interference effects on the modulation properties of liquid crystal displays", Opt. Commun. 265, 84-94 (2006).

26. A. Márquez, M. L. Álvarez, S. Gallego, D. Méndez, E. Fernández, M. Ortuño, A. Beléndez and I. Pascual, "Characterization and optimization of liquid crystal displays for data storage applications", Proc. SPIE 6587 (in press).

27. A. Márquez, C. Cazorla, M. J. Yzuel and J. Campos, "Characterization of the retardance of a wave plate to increase the robustness of amplitude-only and phase-only modulations of a liquid crystal display”, J. Mod. Opt. 52, 633-650 (2005). 